\title{
Administration of simvastatin halts progression of cirrhosis via up-regulating expression of miR-34a and interleukin-10 in rats
}

\section{Type}

Research paper

\section{Keywords}

simvastatin, IL-10, cirrhosis, miR-34a

\begin{abstract}
Introduction

Simvastatin (SIM) treatment has been found to be able to reduce the expression of miR-34a, and we found that interleukin-10 (IL-10) is a potential target gene of miR-34a by searching the online microRNA (miRNA) database. Furthermore, it has been shown that IL10 up-regulation could halt the progression of cirrhosis. The objective of this study was to explore the underlying mechanism of Simvastatin/miR-34a/lL-10 involved in HBV associated cirrhosis.

\section{Material and methods}

Real-time PCR, western-blot analysis, immunohistochemistry, computational analysis, luciferase assay was carried out to explore the underlying mechanism of miR-34a involved in HBV associated cirrhosis.
\end{abstract}

\section{Results}

SIM treatment dose-dependently decreased the levels of miR-34a while increasing the levels of IL-10 mRNA and protein. Levels of IL-10 mRNA and protein were remarkably decreased, while miR-34a mRNA level and active caspase-3 protein level was apparently increased in Cirrhosis group compared with sham group. Accordingly, SIM treatment obstructed the dysregulated miR-34a expression and IL-10 expression in cirrhosis animals. By performing computational analysis, we identified that a complementary binding site of miR-34a was located in IL-10 3' untranslated region (3'UTR), and miR-34a reduced luciferase activity of wild-type IL-10 3'UTR.

\section{Conclusions}

Our data also suggested that SIM may become a new therapeutic strategy for HBV-associated cirrhosis via targeting the miR-34a/IL-10 axis. 
1 Administration of simvastatin halts progression of cirrhosis via up-regulating

2 expression of miR-34a and interleukin-10 in rats

3 Hui Yang ${ }^{1}$, Xiao-rong Zhou ${ }^{2 *}$, Yong-hua Wang ${ }^{1}$, Yan Cheng ${ }^{1}$, Hong-li Zhao ${ }^{1}$, Lu Qiao ${ }^{1}$

4 1. Department of Gastroenterology, The Second Affiliated Hospital of Xi'an Jiaotong $5 \quad$ University, Xi'an, 710004, P.R. China

6 2. Geriatric Surgery, The Second Affiliated Hospital of Xi'an Jiaotong University, Xi'an, 7 710004, P.R. China

$8 \quad *$ Corresponding author: Dr. Xiao-rong Zhou

9 Geriatric Surgery, The Second Affiliated Hospital of Xi'an Jiaotong University, No. 157,

10 Xiwu Road, Xincheng District, Xi'an, Shaanxi, P.R. China

11 Email: surgeondocx@yeah.net

12 Tel: +86-029-87679387

13 Running title: Simvastatin halt cirrhosis via miR-34a/IL-10 


\section{Abstract}

27 Background: Simvastatin (SIM) treatment has been found to be able to reduce the 28 expression of miR-34a, and we found that interleukin-10 (IL-10) is a potential target 29 gene of miR-34a by searching the online microRNA (miRNA) database. Furthermore, it 30 has been shown that IL10 up-regulation could halt the progression of cirrhosis. The 31 objective of this study was to explore the underlying mechanism of Simvastatin/miR32 34a/IL-10 involved in HBV associated cirrhosis. Method: Real-time PCR, western-blot 33 analysis, immunohistochemistry, computational analysis, luciferase assay was carried 34 out to explore the underlying mechanism of miR-34a involved in HBV associated 35 cirrhosis. Results: SIM treatment dose-dependently decreased the levels of miR-34a 36 while increasing the levels of IL-10 mRNA and protein. Levels of IL-10 mRNA and protein 37 were remarkably decreased, while miR-34a mRNA level and active caspase-3 protein 38 level was apparently increased in Cirrhosis group compared with sham group.

39 Accordingly, SIM treatment obstructed the dysregulated miR-34a expression and IL-10

40 expression in cirrhosis animals. By performing computational analysis, we identified that

41 a complementary binding site of miR-34a was located in IL-10 3' untranslated region

42 (3'UTR), and miR-34a reduced luciferase activity of wild-type IL-10 3'UTR. Conclusion:

43 Our data also suggested that SIM may become a new therapeutic strategy for HBV-

44 associated cirrhosis via targeting the miR-34a/IL-10 axis.

45 Key word: Simvastatin, cirrhosis, miR-34a, IL-10

\section{Introduction}

47 Liver cirrhosis has become a significant health burden worldwide. Based on the data

48 released by a 2010 study in Global Burden of Disease, liver cirrhosis has resulted in 31

49 million Disability Adjusted Life Years (DALYs) and 1 million fatalities, accounting for 1.2\%

50 of total DALYs and $2 \%$ of today deaths worldwide [1]. Although extensive efforts have

51 been made to explore the molecular mechanism underlying the development of

52 cirrhosis, few therapeutic agent has been validated in the treatment of the disease [2,3]. 
53 Interleukin-10 (IL-10) has been reported to play an inhibitory role in the development of

54 cirrhosis by decreasing pro-inflammatory responses and regulating hepatic fibrogenesis

55 [4]. In addition, IL-10 was found to exert a direct effect on the synthesis of collagenases

56 and collagen, and hence was involved in the regulation of extracellular matrix (ECM)

57 remodeling [5]. Furthermore, the data from an earlier study showed that IL-10 may be

58 critical to prevent the hepatic fibro genesis induced by carbon tetrachloride $\left(\mathrm{CCl}_{4}\right)[6]$.

59 Moreover, it has been implicated that the IL-10/IL-10 receptor axis could blocked the

60 transcription and protein synthesis of matrix metalloproteinase-2 (MMP-2) in in non-

61 immortalized primary human prostate cell strains derived from prostate cancer [7].

62 As a type of short (18-24 nucleotides in length) and evolutionarily conserved non-coding

63 RNAs, microRNAs (miRNAs) can regulate gene expression at a post-transcriptional level

64 [8]. Studies have demonstrated the involvement of miRNAs in a wide range of biological

65 processes, such as cell differentiation, development and apoptosis [10].

66 A recent study has demonstrated the involvement of several miRNAs in medical

67 condition of liver including cirrhosis [10]. In addition, it was also reported that HCC could

68 be differentiated from cirrhosis by measuring the profile of microRNAs (miRNAs) in the

69 plasma or serum of the patients and the diagnosis and treatment of cirrhosis in an early

70 stage could decrease the incidence of HCC $[14,15]$. Furthermore, Chen et al. used miR-

$71 \quad 181 \mathrm{~b}$ and miR-106b circulating in the plasma as biomarkers to achieve early diagnosis of

72 liver cirrhosis with an area under curve (AUC) value of $0.7 \sim 0.8$ [14]. In another study,

73 up-regulated expression of miR-885-5p was found in the serum of patients suffering

74 from liver cirrhosis (LC) and hepatitis B (HBV). Subsequently, miR-885-5p was used as a

75 candidate biomarker for the diagnosis of cirrhosis [15]. Researchers have found that

76 statins, also known as HMG-CoA reductase inhibitors, are a class of lipid-lowering

77 medications. Statins act to lower cholesterol levels by inhibiting the enzyme HMG-CoA

78 reductase, which is required for cholesterol synthesis. Statins include rosuvastatin,

79 atorvastatin and pitavastatin, and it promotes the progression of non-alcoholic fatty

80 liver disease (NAFLD) by improving the conditions of hepatitis, fibrosis and hepatic

81 steatosis [20, 21]. In addition, simvastatin was found to decrease the abnormally high 
82 level of liver enzymes and to abolish hepatic inflammation, thus stabilizing or reversing

83 the progress of fibrosis by suppressing the proliferation of HSC [18-21]. Simvastatin

84 treatment has been found to be able to reduce the expression of miR-34a, and we

85 found that IL-10 is a potential target gene of miR-34a by searching the online miRNA

86 database. Furthermore, it has been shown that IL-10 up-regulation could halt the

87 progression of cirrhosis in mouse model [22]. Based on the evidence listed above, we

88 tested the regulatory relationship between simvastatin, miR-34a and IL-10, and also

89 investigated the anti-fibrotic effect of simvastatin in rats as well as its potential signaling

90 pathway.

91 Materials and Methods

92 Animal

93 This is an experimental study which has done on the 36 adult female Wistar rats free of

94 specific-pathogen. The rats were obtained from Institutional Animal Center and

95 weighted $250 \pm 20 \mathrm{~g}$. The rats were maintained at $25 \pm 2{ }^{\circ} \mathrm{C}$ along with a schedule of 12 -

96 hour light/dark constantly for 2 weeks to acclimatize. All rats were allowed to water and

97 food. Institutional Ethics Committee had already approved this project. Then equal

98 volume olive oil was utilized to dissolve $\mathrm{CCl}_{4}, 1 \mathrm{~mL} / \mathrm{kg}$ body weight $\mathrm{CCl}_{4}$ was

99 intraperitoneally inject into 24 female rats to induce cirrhosis twice a week, the injection

100 was lasted for 6 weeks. Only olive oil was intraperitoneally injected into 6 female rats to

101 generate normal controls as sham group. Histopathological examination was performed

102 to assess cirrhosis of liver samples. 6 weeks after injection, 24 female rats with cirrhosis

103 were divided into two group: 12 rats with cirrhosis received no treatment as cirrhosis

104 group, other 12 rats with cirrhosis received simvastatin treatment as cirrhosis +

105 simvastain group. $10 \mathrm{mg} / \mathrm{kg}$ body weight simvastatin dissolved in $0.5 \%$ solution of

106 xanthan gum was utilized to treat rats orally every day for 8 weeks. Peripheral blood

107 samples were taken from each rat for future study.

108 RNA isolation and real-time PCR 
SV Total RNA Isolation system (Promega, Madison, WI, USA) was utilized to extract total RNA from HepG2 or LO2 cells following instruction indicated by supplier. Total RNA content was examined using spectrophotometrical analysis at $260 \mathrm{~nm}$. RT-PCR kit

112 (Stratagene USA) was utilized to perform RT-PCR in order to reverse transcribe RNA into 113 IL-10 cDNA with a mixture containing $10 \mu \mathrm{L}$ RNA, $3 \mu \mathrm{L}$ random primers, RNA primer 114 mixture, $1 \mu \mathrm{L} 10 \mathrm{mM}$ deoxynucleotide triphosphates (dNTPs), $1 \mu \mathrm{L}$ RNase inhibitor, $1 \mu \mathrm{L}$ 115 moloneymurine leukemia virus (MMLV)-RT enzyme and $10 \mu \mathrm{L}$ diethylpyrocarbonate 116 (DEPC)-treated water. And the reaction was carried out as follow: $37^{\circ} \mathrm{C}$ for $60 \mathrm{~min}$, then $11795^{\circ} \mathrm{C}$ for $10 \mathrm{~min}$, and followed by cooling at $4{ }^{\circ} \mathrm{C}$. Quantitative real-time RT-PCR was 118 carried to determine the expression of miR-34a using SYBR Premix Ex TaqTM II (Takara, 119 Dalian, China) following supplier's guideline. Real-time RT-PCR was performed to 120 determine IL-10 level using standard SYBR Green RT-PCR Kit (Takara, Otsu, Japan) in 121 accordance with supplier's protocol. Small RNA U6 and GAPDH were served as internal 122 controls for the normalization of miR-34a and IL-10 mRNA respectively. ABI 7500

123 Software 2.04 from Applied Biosystems (Foster City, CA, USA) with 2- $\triangle \triangle C T$ method was 124 utilized to calculate the relative expression of miR-34a and IL-10 mRNA normalized to 125 expressions of U6 and GAPDH. All experiments were repeated in triplicate.

127 HepG2 or LO2 cells were purchased from Chinese Cell Bank of the Chinese Academy of 128 Sciences (Shanghai, China), and DMEM (Dulbecco's modified Eagle's medium) (GIBCO, 129 Carlsbad, CA) supplemented with 10\% FBS (fetal bovine serum) (GIBCO, Carlsbad, CA), $130100 \mathrm{U} / \mathrm{mL}$ penicillin and $100 \mathrm{mg} / \mathrm{mL}$ streptomycin sulfate was utilized to culture cells at $13137^{\circ} \mathrm{C}$ with a atmosphere with $5 \% \mathrm{CO}_{2} .50 \mathrm{nM}$ of miR-34 mimic and its negative control 132 were transfected into HepG2 or LO2 cells using Lipofectamine 2000 (Invitrogen, CA, US) 133 after cells reached $80 \%$ confluence. Meanwhile when the cells reached $80 \%$ confluence, 1341 or 5um simvastatin was utilized to treated HepG2 or LO2 cells for 12 hours. Three 135 independent experiments were carried out.

\section{Cell proliferation assay}


137 HepG2 or LO2 cells were purchased from ATCC and cultured into 12-well plates for 12 138 hours, and then cells transfected with miR-34 mimic or miRNA mimic negative control, 139 and incubated for additional 24 hours to 72 hours, then $10 \mu \mathrm{L} 5 \mathrm{mg} / \mathrm{mL}$ MTT was added 140 into each well, and incubated at $37^{\circ} \mathrm{C}$ for 3 hours with $5 \% \mathrm{CO}_{2}$. Then MTT solution were

141 removed, and $100 \mu \mathrm{L}$ dimethyl sulfoxide (DMSO) (Sigma-Aldrich, St. Louis, MO, USA) was

142 added into each well to dissolve the crystals. A spectrophotometric analysis (BioTek, 143 Grand Island, NY, USA) was utilized to measure cell proliferation at $490 \mathrm{~nm}$. Each test 144 was run in triplicate.

\section{Luciferase assay}

146 PCR was carried out to amplify full fragment wild-type IL-10 3'UTR with putative binding 147 site of miR-34a, above PCR products were then subcloned into $\mathrm{p}$-GL3-control vector 148 (Ambion, Austin, TX, USA) to generate Wt- IL-10-3'UTR. Quick Change Site-Directed 149 Mutagenesis Kit (Agilent, Roseville City, CA) was utilized to obtain mutant IL-10 3'UTR, 150 and also subcloned into same site of pGL3-control vector (Ambion, Austin, TX, USA) to 151 generate Mut- IL-10-3'UTR located downstream of luciferase gene. Then HepG2 or LO2 152 cells were maintained into 24-well plate, Lipofectamine 2000 (Invitrogen, CA, US) was 153 utilized to co-transfect constructs contained wild-type or mutant IL-10 3'UTR and miR15434 a or miR-NC into HepG2 or LO2 cells based on manufacturer's guideline. 48 hours 155 after transfection, Dual-Luciferase Reporter Assay System (Promega, Madison, WI) was 156 utilized to measure luciferase activity of Firefly luciferase and Renilla luciferase. Three 157 independent experiments were carried out.

\section{Western blot analysis}

15948 hours after transfection with miR-34a mimic, ice-cold PBS was utilized to wash the 160 HepG2 or LO2 cells three times, and radioimmuno precipitation assay (Keygen, Nanjing, 161 China) buffer supplemented with $1 \mathrm{mM}$ phenylmethanesulfonylfluoride fluoride 162 (Keygen) was utilized to lyse the cell in accordance with supplier's description. The 163 lysates were subjected to centrifugation at $12000 \times \mathrm{g}$ at $4^{\circ} \mathrm{C}$ for $15 \mathrm{~min}$. BCA protein assay 164 kit (TaKaRa, Japan) was utilized to examine concentration of protein based on 
manufacturer's instruction. 8-12\% SDS-PAGE (Bio-Rad Laboratories, Hertfordshire, UK) was utilized to separate total protein, and then transferred to PVDF (polyvinylidene

167 difluoride) membrane (Immobilon-P; Millipore, Billerica, MA, USA) for 90 min at 120V. $1685 \%$ no-fat milk was utilized to block membrane for $120 \mathrm{~min}$ at room temperature. The 169 primary antibodies against IL-10 ( 1:6,000; Biorbyt Limited, Cambridge, UK) or anti- $\beta$ 170 actin (1:10,000; Biorbyt Limited, Cambridge, UK) was utilized to treat the membrane at $17144^{\circ} \mathrm{C}$ overnight, and TBST (Tris buffered saline with $1 \%$ Tween) was utilized to wash the 172 membrane three times, and HRP (horseradish peroxidase)-labeled secondary antibody 173 (1:15,000; Santa Cruz Biotechnology, Santa Cruz, CA, USA) was utilized to treat the 174 membrane at room temperature for $120 \mathrm{~min}$. Enhanced chemiluminescence (Tanon, 175 Shanghai, China) was utilized to visualize specific bands. All tests were repeated in 176 triplicate.

\section{Apoptosis analysis}

17848 hours following transfection, the cells were collected, and PBS was utilized to wash 179 the cells. FITC-Annexin V/ propidium iodide Apoptosis Detection Kit (BestBio, Shanghai, 180 China) was utilized to treat HepG2 and LO2 cells based on standard guideline indicated 181 by supplier. The flow cytometry (BD FACSCanto II, BD Biosciences, San Jose, USA) was 182 utilized to analyze the data immediately. All reactions was repeated at least three times.

\section{Masson staining}

184 Fontana-Masson kit (Abcam, Cambridge, MA, USA) was utilized to perform Masson 185 staining according to standard protocol. In brief, liver tissues embedded with paraffin 186 were cut into $4 \mu \mathrm{M}$ thickness sections, hexahydro toluene and gradient ethanol were 187 utilized to dewax sections, then rehydrated. Weigert's hematoxylin containing ferric 188 chloride in diluted hydrochloric acid, potassium ferricyanide solution alkalized by 189 sodium borate and hematoxylin in 95\% ethanol was used to stain the nuclei of cells. 190 Then plasma stain supplemented with acid fuchsin, Xylidine Ponceau, glacial acetic acid, 191 and distilled water was utilized to maintain tissue samples. Solution containing 
192 phosphomolybdic acid in distilled water was subsequently utilized to treat tissue

193 samples. Fibre stain with Light Green SF yellowish was used to stain collagen finally.

\section{TUNEL}

195 TUNEL assay was utilized to examine apoptosis of sections. Briefly, $50 \mu \mathrm{L}$ TUNEL reaction 196 buffer (Roche Applied Science, Bael, Swiss) was utilized to treat sections in the darkness 197 at $37^{\circ} \mathrm{C}$ for 1 hour in a humidified atmosphere. DAPI was utilized to stain cell nuclei by 198 maintaining with the sections at RT in the darkness for $5 \mathrm{~min}$. Then flow cytometry was 199 utilized to determine number of positive cells. The ratio was calculated as number of 200 TUNEL positive cells /total cells.

\section{Statistical analysis}

202 All data were shown as mean \pm SD (standard deviation). SPSS software version 16.0 203 (SPSS Inc., Chicago, IL, USA) was utilized to perform statistical analysis. Independent t204 test was utilized to analyze comparisons of continuous data, $\chi 2$ test was utilized to 205 analyze categorical data. P value less than 0.05 was considered to be statistically 206 significant.

207 Results

208 IL-10 is a candidate gene of miR-34a

209 Bioinformatics algorithms including TargetScan were utilized to predict miR-34a target 210 gene. Based on the results of algorithms above, we predicted IL-10 might be a possible 211 target gene of miR-34a with a complementary seed region of miR-34a (Fig. 1A). To

212 further confirm IL-10 is a candidate gene of miR-34a, we then conducted luciferase 213 assay, and sub-cloned wild or mutant IL-10 3'UTR into luciferase reporter which located

214 direct downstream of luciferase gene. Then HepG2 and LO2 cells co-transfected with

215 luciferase reporter carried wild or mutant IL-10 3'UTR and different dose of miR-34a.

216 Luciferase activity of wild-type IL-10 3'UTR in HepG2 (Fig. 1B) and LO2 (Fig. 1D) cells

217 showed a stepwise decline as the concentration of miR-34a mimics increased when

218 compared with the negative controls, and miR-34a had no effect on luciferase activity of 
mutant IL-10 3'UTR in HepG2 (Fig. 1C) and LO2 (Fig. 1E) cells, indicating that miR-34a directly targeted IL-10.

\section{Effect of simvastatin on transcription activity of miR-34a promoter}

222 To further confirm whether simvastatin affected transcription activity of miR-34a

223 promoter, we then conducted luciferase assay, and sub-cloned miR-34a promoter into

224 luciferase reporter which located direct upstream of luciferase gene (Fig. 2A), then

225 different dose of simvastatin was utilized to treated HepG2 and HepG2 cells transfected 226 with constructs containing miR-34a promoter. As shown in Fig. 2, luciferase activity of 227 miR-34a promoter in HepG2 (Fig. 2B) and LO2 (Fig. 2C) cells showed a stepwise decline 228 as the concentration of simvastatin increased when compared with the negative 229 controls, suggesting that simvastatin inhibited transcription activity of miR-34a 230 promoter.

\section{Effect of simvastatin on miR-34a and IL-10 levels}

232 Real-time PCR and western-blot analysis were utilized to examine levels of miR-34a and 233 IL-10 in HepG2 and LO2 cells treated with different dose of simvastatin. As shown in Fig.

234 3, miR-34a level in HepG2 (Fig.3A) and LO2 (Fig. 3E) cells treated with simvastatin was 235 reduced compared with control under a dose-dependent manner, while simvastatin 236 dose-dependently enhanced IL-10 mRNA (Fig. 3B and 3F) and protein (Fig. 3C, 3D, 3G, $2373 \mathrm{H}$ ) expressions in HepG2 (Fig. 3A-D) and LO2 (Fig. 3E-H) cells compared with control.

\section{MiR-34a and IL-10 varied among different groups}

239 All animals were divided into three groups: sham, cirrhosis and cirrhosis treated with 240 simvastatin. And miR-34a and IL-10 among above three groups using real-time PCR and 241 western-blot analysis. As shown in Fig. 4A, animals diagnosed with cirrhosis evidently 242 increased miR-34a compared with sham group, while administration of simvastatin 243 partially restored expression of miR-34a. IL-10 mRNA (Fig. 4B) and protein (Fig. 4C) 244 levels in cirrhosis group were much lower than cirrhosis + simvastatin group, both of 245 them were much lower than sham group. Also, animals diagnosed with cirrhosis 
246 evidently increased caspase-3 level compared with sham group, while administration of

247 simvastatin partially restored expression of caspase-3. Masson staining was performed

248 to detect degree of cirrhosis among sham, cirrhosis and cirrhosis+ simvastatin groups.

249 As shown in Fig. 4E, degree of cirrhosis in cirrhosis group were much higher than

250 cirrhosis + simvastatin group, both of them were much higher than sham group.

\section{Differential apoptosis among various groups}

252 TUNEL assay was performed to determine apoptosis among sham, cirrhosis and

253 cirrhosis+ simvastatin groups. As shown in Fig. 4F, apoptosis in cirrhosis group was much

254 higher than cirrhosis + simvastain group, both of them were much higher than sham

255 group.

256 Discussion

257 Using rodent cirrhosis models, several preclinical trials have demonstrated the potential 258 advantages of statins to treat portal hypertension [23]. Furthermore, a pilot study on

259 cirrhosis patients suggested that one single dose of simvastatin given by oral

260 administration could result in a sharp decline in vascular resistance of the liver [24].

261 Subsequently, a randomized and placebo-controlled multicenter study demonstrated

262 that a one-month treatment with simvastatin led to a decreased portal pressure and

263 improved clearance of indocyanine green, suggesting the improvement in liver functions

264 [25]. Overall, these data indicated that, in the cirrhosis patients suffering from acute

265 variceal bleeding, simvastatin may ameliorate the prognosis of these patients by

266 influencing the two key factors involved, i.e., liver functions and portal pressure. In

267 particular, a recent clinical trial also obtained similar data demonstrating that

268 simvastatin acted as a liver-selective and potent vasodilator, since the systemic vascular

269 resistance and average arterial pressure remained stable after the prolonged

270 administration of simvastatin [25]. In this study, we detected levels of miR-34a, IL-10

271 mRNA and protein level among the rat model groups using real-time PCR and western-

272 blot analysis, and validated that miR-34a level in cirrhosis group was much higher than

273 cirrhosis + simvastatin group, both of them were much higher than sham group. 
274 However, IL-10 expression in cirrhosis group was much lower than cirrhosis +

275 simvastatin group, both of them were much lower than sham group. Moreover, we

276 performed TUNEL assay to detect apoptosis among sham, cirrhosis and cirrhosis+

277 simvastatin groups, and found that apoptosis in cirrhosis group was much higher than

278 cirrhosis + simvastatin group, both of them were much higher than sham group.

279 A previous study has shown that, via the suppression of miR-34a expression, the 280 application of atorvastatin could benefit endothelial functions by increasing the 281 expression of SIRT1 [21]. Furthermore, during the progression of NAFLD, the levels of 282 apoptosis, acetylated p53 and microRNA-34a in the fibrotic tissues of the liver were all 283 gradually elevated [30]. These results suggested that statins, especially simvastatin, may 284 play a critical role in improving hepatic inflammation, steatosis and fibrosis by regulating 285 the pathways related to microRNA-34a [27]. In human patients or animal models of 286 NAFLD, cirrhosis, alcoholic liver injury and HCC, the expression of miR-34a was elevated 287 and was dependant on the severity of the disease [28]. In addition, miR-34a was found 288 as a direct target of p53. Since sirtuin 1 (SIRT1) acts as the target gene of miR-34a and 289 can suppress p53-dependent apoptosis via the deacetylation of all major sites involved 290 in p53 acetylation, a positive loop of feedback is formed along the SIRT1/miR-34a/p53 291 signaling pathway, which in turn plays a critical role in regulating the apoptosis and 292 proliferation of cells [29, 30]. It was also shown that, by targeting IL-10, miR-34 exerted 293 an indirect effect on the induction of MDSC [31]. In this study, we carried out 294 computational analysis to predict miR-34a target gene, and identified that IL-10 as a 295 virtual target gene of miR-34a, and luciferase activity of wild-type IL-10 3'UTR was dose296 dependently reduced by transfecting with miR-34a. In addition, we investigate effect of 297 simvastatin on transcription activity of miR-34a promoter using luciferase assay, and 298 found that simvastatin inhibited luciferase activity droved by miR-34a promoter under a 299 dose-dependent manner.

300 As an anti-inflammatory cytokine cytokine, interleukin-10 (IL-10) is synthesized by 301 monocytes/macrophages, regulatory T cells and Th2 cells. IL-10 can inhibit the 302 production of cytokines, including IFN $\gamma$ from $T$ cells as well as TNF- $\alpha, I L-1 \alpha, I L-16$ and IL-6 
from activated macrophages [32]. Located on chromosome 1 (1q31-1q32), the gene encoding $I L-10$ is about $4.7 \mathrm{~kb}$ and includes five exons and four introns [33]. A few studies have investigated the effect of IL-10 gene polymorphisms on the susceptibility to liver cirrhosis, although their conclusions were inconsistent [34]. For example, it was shown that the rs1800896 polymorphism of IL-10 was associated with the elevated risk of liver cirrhosis, particularly in patients suffering from chronic infection of hepatitis $B$ [35]. In this study, we performed real-time PCR and western-blot analysis to detect influence of simvastatin on expressions of miR-34a and IL-10, and revealed that simvastatin decreased miR-34a level under a dose-dependent manner, while enhanced IL-10 expression under a dose-dependent manner.

As an important and pleiotropic cytokine with immunoregulatory features, interleukin 10 (IL-10) is mainly produced in macrophages, although it can also be synthesized in mast cells, monocytes, dendritic cells, B lymphocytes, cytotoxic T cells, as well as in T helper 1 (Th1) cells, Th2 cells and even in human carcinoma cell lines [36]. The activity of IL-10 is regulated by IL-10 receptor (IL-10R), a member of the class II cytokine receptor family. By inhibiting the expression of major histocompatibility complex (MHC) class II and co-stimulatory factors including CD80 (B7.1) and CD86 (B7.2), IL-10 reduces the ability of macrophages and monocytes to present antigens to T cells, thus reducing the

322 it was found that IL-10 could prevent the apoptosis of B cells and enhance their

323 proliferation, thus playing a role of immunoglobulin (Ig) class switch.

324 As a cytokine that reduces pro-inflammatory responses and regulates hepatic

325 fibrogenesis, IL-10 may provide a therapeutic alternative for patients with HCV-related

326 cirrhosis that do not respond to IFN-based therapy [37, 38]. In addition, IL-10 was found

327 to ameliorate fibrosis by suppressing the activity of HSC [39]. Similar results were also

328 obtained using a rat model, in which the administration of exogenous IL-10 reversed

$329 \mathrm{CCl}_{4}$-induced fibrosis in the liver by decreasing the expression of TIMP-1 and TGF- $\beta 1$ 330 [37].

\section{Conclusion}


332 In our study, we suggested that administration of simvastatin halts progression of

333 cirrhosis via up-regulating expression of miR-34a and interleukin-10 in rats. In brief, we

334 found that Simvastatin treatment has been found to be able to reduce the expression of

335 miR-34a, and we found that IL-10 is a potential target gene of miR-34a by searching the

336 online miRNA database. Furthermore, we revealed that IL-10 up-regulation could halt

337 the progression of cirrhosis in mouse model.

\section{Conflict of interest}

339 None

\section{Acknowledgements}

$341 \quad$ None.

\section{References}

3431 Murray CJ, Vos T, Lozano R, Naghavi M, Flaxman AD, Michaud C, et al.: Disability344 adjusted life years (DALYs) for 291 diseases and injuries in 21 regions, 1990-

345 2010: a systematic analysis for the Global Burden of Disease Study 2010. Lancet $346 \quad 2012 ; 380: 2197-2223$.

3472 Bosetti C, Levi F, Lucchini F, Zatonski WA, Negri E, La Vecchia C: Worldwide 348 mortality from cirrhosis: an update to 2002. J Hepatol 2007;46:827-839.

3493 Rani M, Yang B, Nesbit R: Hepatitis B control by 2012 in the WHO Western Pacific 350 Region: rationale and implications. Bull World Health Organ 2009;87:707-713.

3514 Farkas Z, Rashid T, Chen Y, Siddiqui T, Yandrapalli S,Frager S, Aronow W, Bodin R, 352 Maddineni S: The correlation between sarcopaenia and post-transjugular 353 intrahepatic portosystemic shunt hepatic encephalopathy: a single-institution 354 review. Archives of Medical Science - Atherosclerotic Diseases. 2019;4(1):89-93.

3555 Louis H, Le Moine O, Goldman M, Deviere J: Modulation of liver injury by 356 interleukin-10. Acta Gastroenterol Belg 2003;66:7-14. 
3576 Huang YH, Shi MN, Zheng WD, Zhang $\amalg$, Chen ZX, Wang XZ: Therapeutic effect of 358 interleukin-10 on CCl4-induced hepatic fibrosis in rats. World J Gastroenterol $359 \quad 2006 ; 12: 1386-1391$.

3607 Stearns ME, Wang M, Hu Y, Garcia FU, Rhim J: Interleukin 10 Blocks Matrix 361 Metalloproteinase-2 and Membrane Type 1-Matrix Metalloproteinase Synthesis in Primary Human Prostate Tumor Lines. Clin Can Res 2013; 9(3).

3638 Bartel DP: MicroRNAs: genomics, biogenesis, mechanism, and function. Cell $364 \quad 2004 ; 116: 281-297$.

3659 He L, Hannon GJ: MicroRNAs: small RNAs with a big role in gene regulation. Nat $366 \quad$ Rev Genet 2004;5:522-531.

36710 Budhu A, Jia HL, Forgues M, Liu CG, Goldstein D, Lam A, Zanetti KA, Ye QH, Qin 368 LX, Croce CM, Tang ZY, Wang XW: Identification of metastasis-related microRNAs 369

11 Qi P, Cheng SQ, Wang H, Li N, Chen YF, Gao CF: Serum microRNAs as biomarkers for hepatocellular carcinoma in Chinese patients with chronic hepatitis B virus infection. PLoS One 2011;6:e28486.

37312 Bolondi L, Sofia S, Siringo S, Gaiani S, Casali A, Zironi G, Piscaglia F, Gramantieri L, 374 Zanetti M, Sherman M: Surveillance programme of cirrhotic patients for early 375 diagnosis and treatment of hepatocellular carcinoma: a cost effectiveness $376 \quad$ analysis. Gut 2001;48:251-259.

37713 Xiong J, Zhang M, Guo X, Pu L, Xiong H, Xiang P, Liu J, Li A: Acute kidney injury in $378 \quad$ critically ill cirrhotic patients with spontaneous bacterial peritonitis: a comparison of KDIGO and ICA criteria. Arch Med Sci 2020;16(3):569-576.

14 Chen YJ, Zhu JM, Wu H, Fan J, Zhou J, Hu J, Yu Q, Liu TT, Yang L, Wu CL, Guo XL, Huang XW, Shen XZ: Circulating microRNAs as a Fingerprint for Liver Cirrhosis. PLoS One 2013;8:e66577. 
15 Gui J, Tian Y, Wen X, Zhang W, Zhang P, Gao J, Run W, Tian L, Jia X, Gao Y: Serum microRNA characterization identifies miR-885-5p as a potential marker for detecting liver pathologies. Clin Sci (Lond) 2011;120:183-193.

16 Miyaki T, Nojiri S, Shinkai N, Kusakabe A, Matsuura K, lio E, Takahashi S, Yan G, Ikeda K, Joh T: Pitavastatin inhibits hepatic steatosis and fibrosis in non-alcoholic steatohepatitis model rats. Hepatol Res 2011;41:375-385.

17 Socha M, Pietrzak A, Grywalska E, Pietrzak D, Matosiuk D, Kiciński P, Rolinski J: The effect of statins on psoriasis severity: a meta-analysis of randomized clinical trials. Arch Med Sci 2020;16(1):1-7.

18 Abel T, Feher J, Dinya E, Eldin MG, Kovacs A: Safety and efficacy of combined ezetimibe/simvastatin treatment and simvastatin monotherapy in patients with non-alcoholic fatty liver disease. Med Sci Monit 2009;15:MS6-11.

19 Ekstedt M, Franzen LE, Mathiesen UL, Holmqvist M, Bodemar G, Kechagias S: Statins in non-alcoholic fatty liver disease and chronically elevated liver enzymes: a histopathological follow-up study. J Hepatol 2007;47:135-141.

20 Rombouts K, Kisanga E, Hellemans K, Wielant A, Schuppan D, Geerts A: Effect of HMG-CoA reductase inhibitors on proliferation and protein synthesis by rat hepatic stellate cells. J Hepatol 2003;38:564-572.

21 Tabuchi T, Satoh M, Itoh T, Nakamura M: MicroRNA-34a regulates the longevityassociated protein SIRT1 in coronary artery disease: effect of statins on SIRT1 and microRNA-34a expression. Clin Sci (Lond) 2012;123:161-171.

22 Elmasry A, Aladeeb NM, Elkaref A, Aboulfotouh N: Simvastatin exerts antifibrotic effect and potentiates the antischistosomal effects of praziquantel in a murine model: Role of IL10. Biomed Pharmacother 2017;96:215-221. 
23 Abraldes JG, Rodriguez-Vilarrupla A, Graupera M, Zafra C, Garcia-Caldero H, Garcia-Pagan JC, Bosch J: Simvastatin treatment improves liver sinusoidal endothelial dysfunction in CCl4 cirrhotic rats. J Hepatol 2007;46:1040-1046.

24 Zafra C, Abraldes JG, Turnes J, Berzigotti A, Fernandez M, Garca-Pagan JC, Rodes J, Bosch J: Simvastatin enhances hepatic nitric oxide production and decreases the hepatic vascular tone in patients with cirrhosis. Gastroenterology 2004;126:749-755.

25 Abraldes JG, Albillos A, Banares R, Turnes J, Gonzalez R, Garcia-Pagan JC, Bosch J: Simvastatin lowers portal pressure in patients with cirrhosis and portal hypertension: a randomized controlled trial. Gastroenterology 2009;136:16511658.

26 Castro RE, Ferreira DM, Afonso MB, Borralho PM, Machado MV, Cortez-Pinto H, Rodrigues CM: miR-34a/SIRT1/p53 is suppressed by ursodeoxycholic acid in the rat liver and activated by disease severity in human non-alcoholic fatty liver disease. J Hepatol 2013;58:119-125.

27 Wang W, Zhao C, Zhou J, Zhen Z, Wang Y, Shen C: Simvastatin ameliorates liver fibrosis via mediating nitric oxide synthase in rats with non-alcoholic steatohepatitis-related liver fibrosis. PLoS One 2013;8:e76538.

28 Meng F, Glaser SS, Francis H, Yang F, Han Y, Stokes A, Staloch D, McCarra J, Liu J, Venter J, Zhao H, Liu X, Francis T, Swendsen S, Liu CG, Tsukamoto H, Alpini G: Epigenetic regulation of miR-34a expression in alcoholic liver injury. Am J Pathol 2012;181:804-817.

29 He L, He X, Lim LP, de Stanchina E, Xuan Z, Liang Y, Xue W, Zender L, Magnus J, Ridzon D, Jackson AL, Linsley PS, Chen C, Lowe SW, Cleary MA, Hannon GJ: A microRNA component of the p53 tumour suppressor network. Nature 2007;447:1130-1134. 
43330 Yamakuchi M, Lowenstein CJ: MiR-34, SIRT1 and p53: the feedback loop. Cell $434 \quad$ Cycle 2009;8:712-715.

31 Wang $X$, Chang X, Zhuo G, Sun M, Yin K: Twist and miR-34a are involved in the generation of tumor-educated myeloid-derived suppressor cells. Int J Mol Sci 2013;14:20459-20477.

32 D'Andrea A, Aste-Amezaga M, Valiante NM, Ma X, Kubin M, Trinchieri G: Interleukin 10 (IL-10) inhibits human lymphocyte interferon gamma-production by suppressing natural killer cell stimulatory factor/IL-12 synthesis in accessory cells. J Exp Med 1993;178:1041-1048.

33 Damgaard Sandahl T: Alcoholic hepatitis. Dan Med J 2014;61:B4755.

34 Liu Y, Yu MC, Zhang AQ, Wang YB, Jiang K, Dong JH: Interleukin-10 gene promoter polymorphism and risk of liver cirrhosis. Genet Mol Res 2015;14:12291234.

35 Yao L, Xing S, Fu X, Song H, Wang Z, Tang J, Zhao Y: Association between interleukin-10 gene promoter polymorphisms and susceptibility to liver cirrhosis. Int J Clin Exp Pathol 2015;8:11680-11684.

36 Gastl GA, Abrams JS, Nanus DM, Oosterkamp R, Silver J, Liu F, Chen M, Albino AP, Bander NH: Interleukin-10 production by human carcinoma cell lines and its relationship to interleukin-6 expression. Int J Cancer 1993;55:96-101.

37 Chou WY, Lu CN, Lee TH, Wu CL, Hung KS, Concejero AM, Jawan B, Wang CH: Electroporative interleukin-10 gene transfer ameliorates carbon tetrachlorideinduced murine liver fibrosis by MMP and TIMP modulation. Acta Pharmacol Sin 2006;27:469-476.

38 Nelson DR, Lauwers GY, Lau JY, Davis GL: Interleukin 10 treatment reduces fibrosis in patients with chronic hepatitis C: a pilot trial of interferon nonresponders. Gastroenterology 2000;118:655-660. 
39 Zhang $\amalg$, Zheng WD, Chen YX, Huang YH, Chen ZX, Zhang SJ, Shi MN, Wang XZ: Antifibrotic effects of interleukin-10 on experimental hepatic fibrosis. Hepatogastroenterology 2007;54:2092-2098.

462 Figure legends

\section{$463 \quad$ Figure 1}

464 IL-10 is confirmed as a candidate gene of miR-34a by computational analysis and 465 luciferase assay (WT: wild-type; MUT: mutant; N=3; * P value < 0.05 vs. 0 group).

466 A: Comparison between miR-34a and wild-type/mutant IL-10 3'UTR.

467 B: Luciferase activity of wild-type IL-10 3'UTR was inhibited under a dose-dependent 468 manner in HepG2 cells

469 C: Luciferase activity of wild-type IL-10 3'UTR was dose-dependently decreased under a 470 dose-dependent manner in LO2 cells

471 D: MiR-34a had no effect on luciferase activity of mutant IL-10 3'UTR in HepG2 cells

472 E: MiR-34a had no effect on luciferase activity of mutant IL-10 3'UTR in LO2 cells

\section{$473 \quad$ Figure 2}

474 Effect of simvastatin on transcription activity of miR-34a promoter detected by 475 luciferase assay (SIM: simvastatin; $N=3 ; *$ P value $<0.05$ vs. untreated group).

476 A: MiR-34a promoter was inserted into luciferase reporter which located direct 477 upstream of luciferase gene

478 B: Luciferase activity droved by miR-34a promoter in HepG2 cells showed a stepwise 479 decline as the concentration of simvastatin increased when compared with the negative 480 controls

481 C: Luciferase activity droved by miR-34a promoter in LO2 cells showed a stepwise 482 decline as the concentration of simvastatin increased when compared with the negative 483 controls 


\section{$484 \quad$ Figure 3}

485 Simvastatin varied expressions of miR-34a and IL-10 in HepG2 and LO2 cells detected by 486 real-time PCR and Western-blot analysis (SIM: simvastatin; $N=3$; * P value $<0.05$ vs.

487 untreated group).

488 A: Simvastatin dose-dependently inhibited miR-34a expression

489 B: IL-10 mRNA level was increased subsequent to treat with simvastatin under a dose-

490 dependent manner

491 C: IL-10 protein expression was enhanced subsequent to treat with simvastatin under a 492 dose-dependent manner

493 D: IL-10 protein expression was enhanced subsequent to treat with simvastatin under a 494 dose-dependent manner

495 E: Simvastatin dose-dependently inhibited miR-34a expression

496 F: IL-10 mRNA level was increased subsequent to treat with simvastatin under a dose-

497 dependent manner

498 G: IL-10 protein expression was enhanced subsequent to treat with simvastatin under a 499 dose-dependent manner

$500 \mathrm{H}$ : IL-10 protein expression was enhanced subsequent to treat with simvastatin under a 501 dose-dependent manner

\section{Figure 4}

503 Differential levels of miR-34a and IL-10, degree of cirrhosis and apoptosis status among 504 different groups detected by real-time PRC, Western-blot analysis, Masson staining and 505 TUNEL assay (Sham: sham-operated; N=3; * P value < 0.05 vs. sham group; ** P value < 5060.05 vs. Cirrhosis group).

507 A: MiR-34a level in cirrhosis + simvastatin group was much higher than sham group, 508 which was even higher in cirrhosis group than cirrhosis + simvastatin group 
509 B: IL-10 mRNA in cirrhosis + simvastatin group was much lower than sham group, which 510 was even lower in cirrhosis group than cirrhosis + simvastatin group

511 C: IL-10 protein in cirrhosis + simvastatin group was much lower than sham group, which 512 was even lower in cirrhosis group than cirrhosis + simvastatin group

513 D: Capase-3 protein in cirrhosis + simvastatin group was much higher than sham group, 514 which was even higher in cirrhosis group than cirrhosis + simvastatin group

515 E: Degree of cirrhosis in cirrhosis group were much higher than cirrhosis + simvastatin 516 group, both of them were much higher than sham group

517 F: Apoptosis in cirrhosis group was much higher than cirrhosis + simvastatin group, both 518 of them were much higher than sham group. 
A



B



D

C

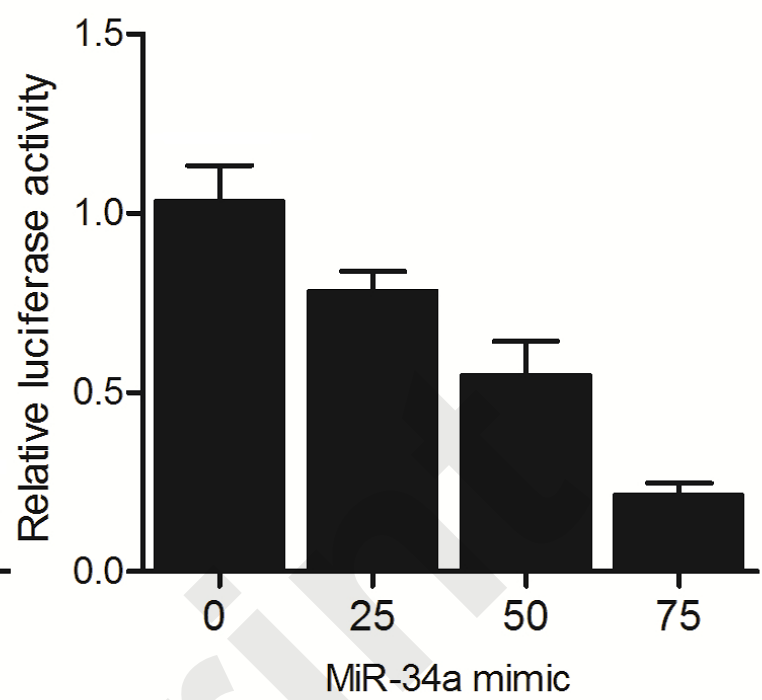

E


Figure 1

IL-10 is confirmed as a candidate gene of miR-34a by computational analysis and luciferase assay (WT: wild-type; MUT: mutant; $N=3$; ${ }^{*} \mathrm{P}$ value $<0.05$ vs. 0 group).

A: Comparison between miR-34a and wild-type/mutant IL-10 3'UTR.

B: Luciferase activity of wild-type IL-10 3'UTR was inhibited under a dose-dependent manner in HepG2 cells

C: Luciferase activity of wild-type IL-10 3'UTR was dose-dependently decreased under a dose-dependent manner in LO2 cells

D: MiR-34a had no effect on luciferase activity of mutant IL-10 3'UTR in HepG2 cells 
E: MiR-34a had no effect on luciferase activity of mutant IL-10 3'UTR in LO2 cells 
A



B

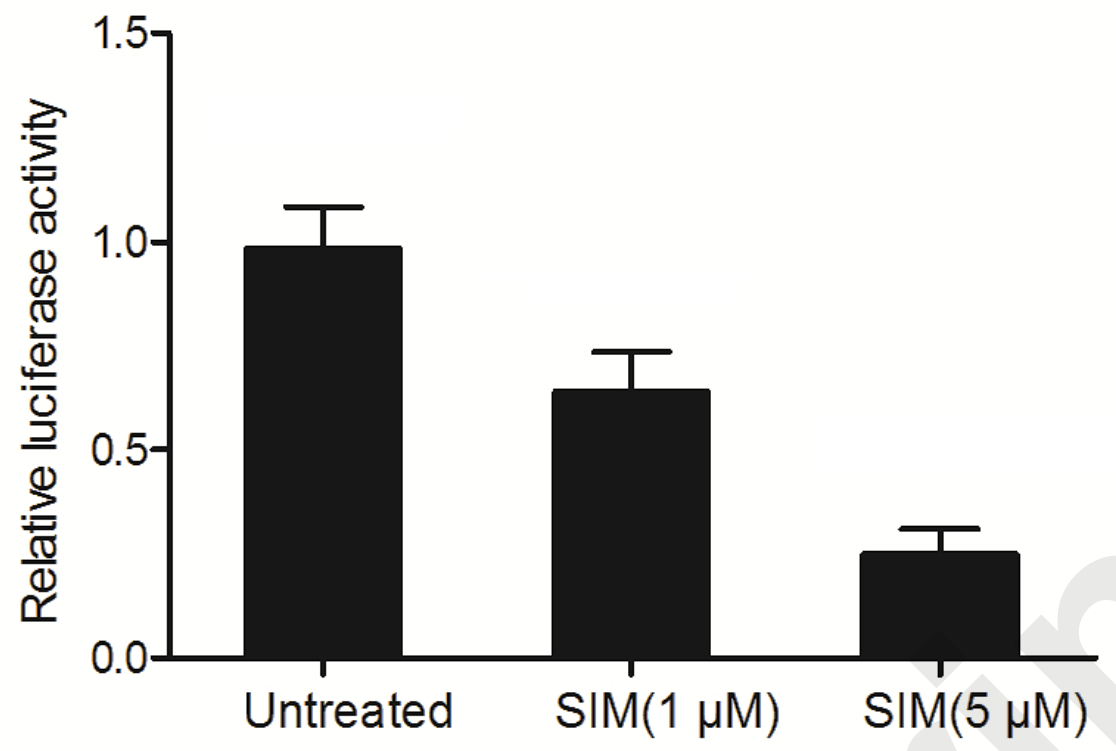

C

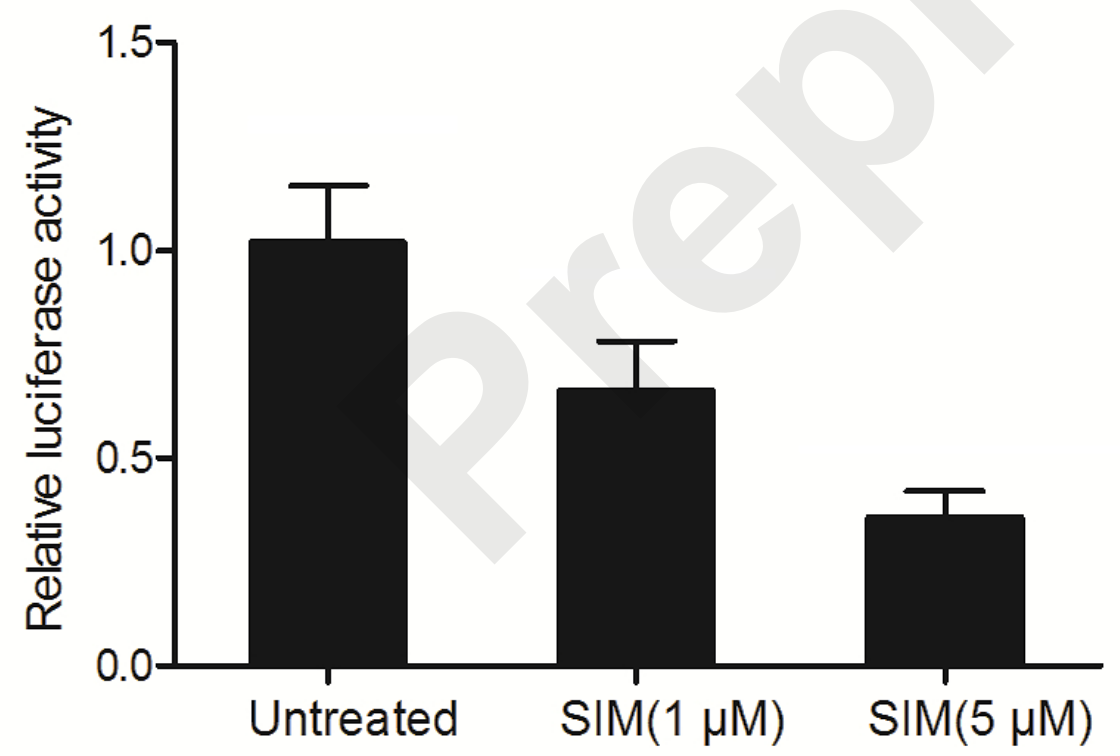

Figure 2

Effect of simvastatin on transcription activity of miR-34a promoter detected by luciferase assay (SIM: simvastatin; $N=3$; * $P$ value $<0.05$ vs. untreated group).

A: MiR-34a promoter was inserted into luciferase reporter which located direct upstream of luciferase gene

B: Luciferase activity droved by miR-34a promoter in HepG2 cells showed a stepwise decline as the concentration of simvastatin increased when compared with the negative controls

C: Luciferase activity droved by miR-34a promoter in LO2 cells showed a stepwise decline 
as the concentration of simvastatin increased when compared with the negative controls 
A

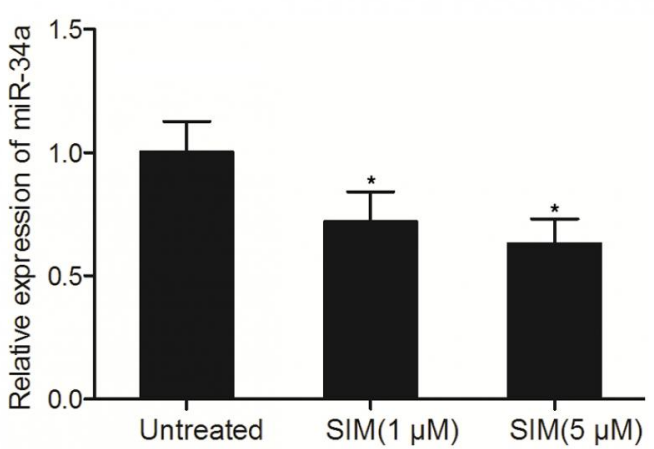

B

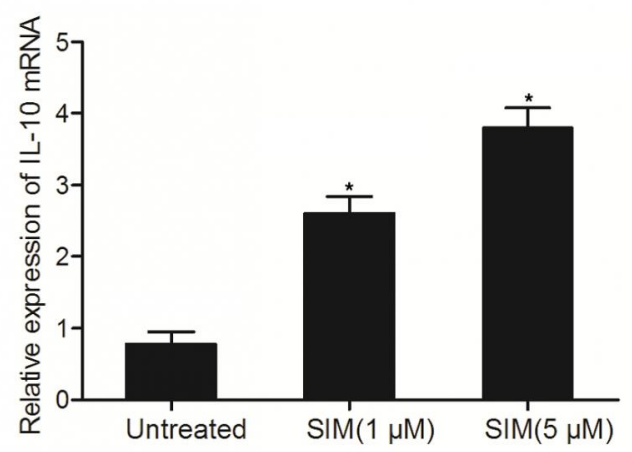

C

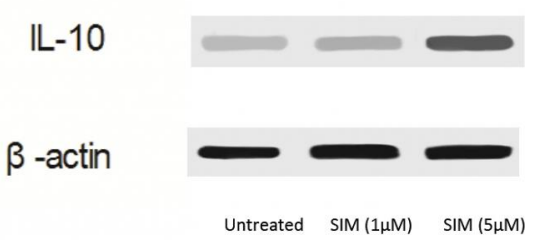

D

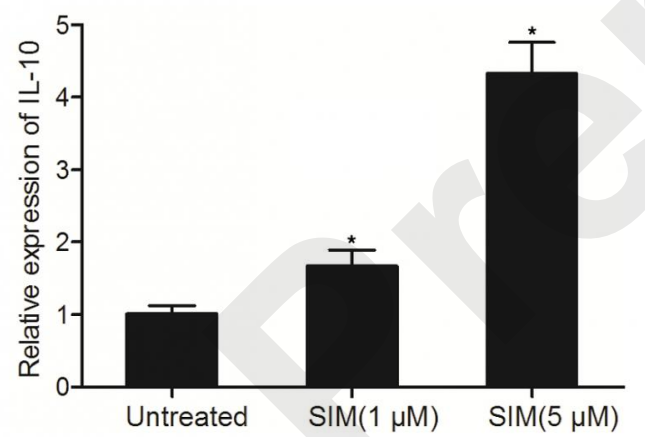

$\mathbf{E}$

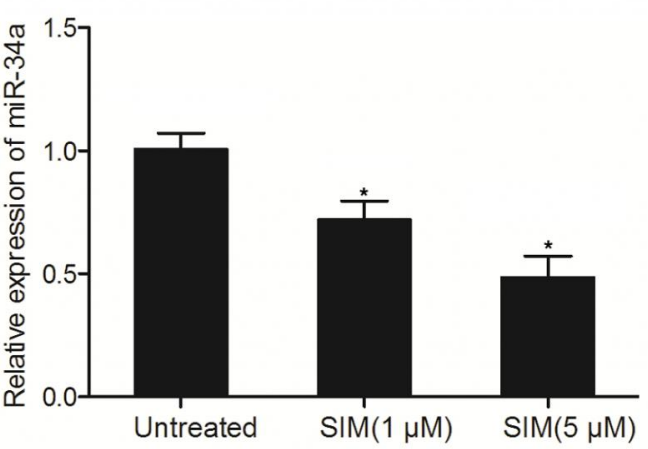

$\mathbf{F}$

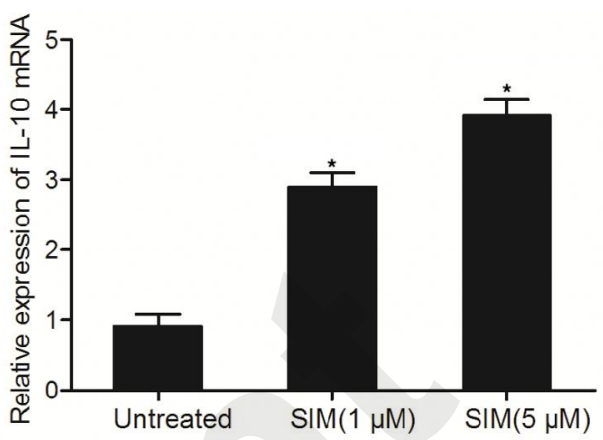

G



\section{$\beta$-actin}

Untreated $\quad \operatorname{SIM}(1 \mu \mathrm{M}) \quad \operatorname{SIM}(5 \mu \mathrm{M})$

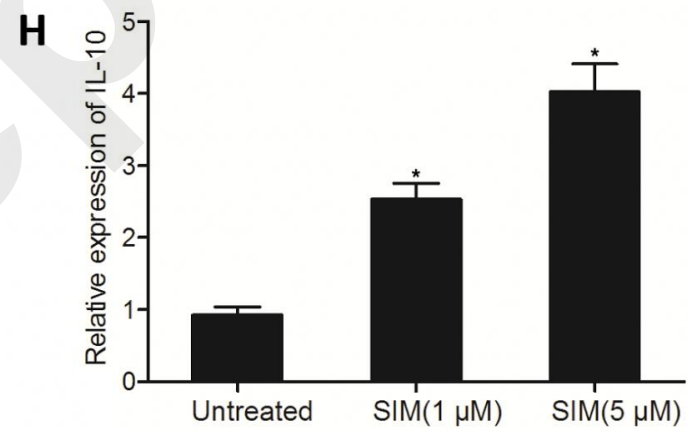

Figure 3

Simvastatin varied expressions of miR-34a and IL-10 in HepG2 and LO2 cells detected by real-time PCR and Western-blot analysis (SIM: simvastatin; $N=3$; * $P$ value $<0.05$ vs. untreated group).

A: Simvastatin dose-dependently inhibited miR-34a expression

$\mathrm{B}$ : IL-10 mRNA level was increased subsequent to treat with simvastatin under a dosedependent manner

C: IL-10 protein expression was enhanced subsequent to treat with simvastatin under a dose-dependent manner

D: IL-10 protein expression was enhanced subsequent to treat with simvastatin under a dose-dependent manner

E: Simvastatin dose-dependently inhibited miR-34a expression

F: IL-10 mRNA level was increased subsequent to treat with simvastatin under a dose- 
dependent manner

G: IL-10 protein expression was enhanced subsequent to treat with simvastatin under a dose-dependent manner

$\mathrm{H}$ : IL-10 protein expression was enhanced subsequent to treat with simvastatin under a dose-dependent manner 
A
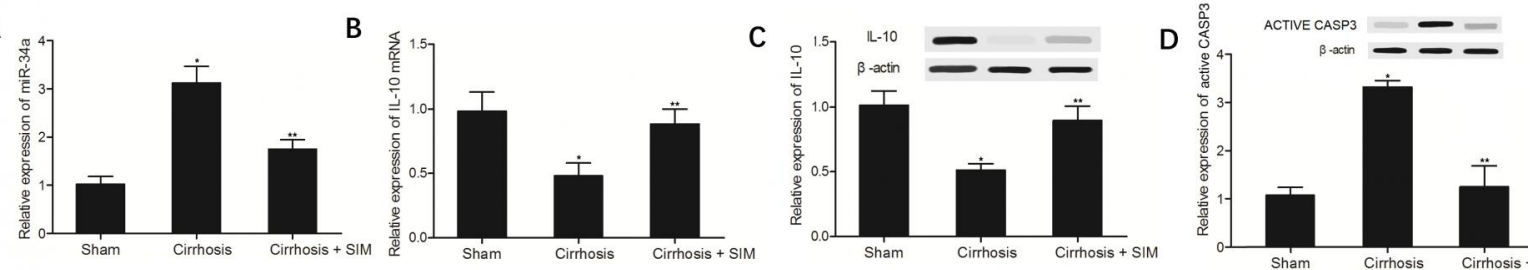

E

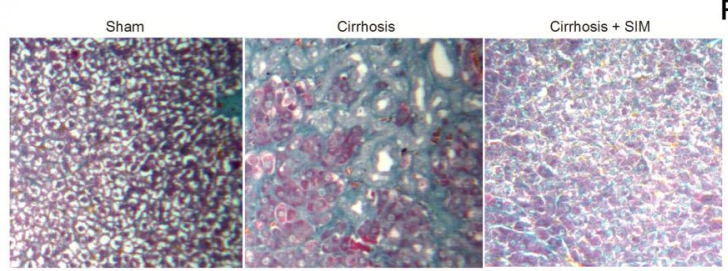

$\mathbf{F}$

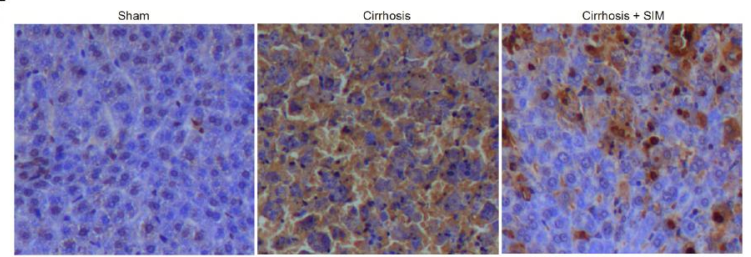

Figure 4

Differential levels of miR-34a and IL-10, degree of cirrhosis and apoptosis status among different groups detected by real-time PRC, Western-blot analysis, Masson staining and TUNEL assay (Sham: sham-operated; $N=3$; ${ }^{*} \mathrm{P}$ value $<0.05$ vs. sham group; ${ }^{* *} \mathrm{P}$ value $<$ 0.05 vs. Cirrhosis group).

A: MiR-34a level in cirrhosis + simvastatin group was much higher than sham group, which was even higher in cirrhosis group than cirrhosis + simvastatin group

B: IL-10 mRNA in cirrhosis + simvastatin group was much lower than sham group, which was even lower in cirrhosis group than cirrhosis + simvastatin group

C: IL-10 protein in cirrhosis + simvastatin group was much lower than sham group, which was even lower in cirrhosis group than cirrhosis + simvastatin group

D: Capase-3 protein in cirrhosis + simvastatin group was much higher than sham group, which was even higher in cirrhosis group than cirrhosis + simvastatin group

$\mathrm{E}$ : Degree of cirrhosis in cirrhosis group were much higher than cirrhosis + simvastatin group, both of them were much higher than sham group

F: Apoptosis in cirrhosis group was much higher than cirrhosis + simvastatin group, both of them were much higher than sham group. 